\title{
N WAVE AND PERIODIC WAVE SOLUTIONS FOR BURGERS EQUATIONS
}

\section{ZAHIA NOURI, SAIDA BENDAAS* AND HOUSSEM EDDINE KADEM}

Département de Mathématiques, Faculté des sciences, Université Ferhat Abbas, Sétif 1. Campus El-Bez, 19000, Sétif, Algérie

${ }^{*}$ Corresponding author: saida_bendaas@yahoo.fr

\begin{abstract}
This article concerns the initial boundary value problem for the non linear dissipative Burgers equation. Our general purpose is to describe the asymptotic behavior of the solution in the Cauchy problem with a small parameter $\varepsilon$ for this equation and to discuss in particular the cases of the $\mathrm{N}$ wave shock and periodic wave shock. we show that the solution of Cauchy problem of viscid equation approach the shock type solution for the Cauchy problem of the inviscid equation for each case. The results are formulated in classical mathematics and proved with infinitesimal techniques of Non Standard Analysis.
\end{abstract}

\section{INTRODUCTION}

Burgers equation is the scalar partial differential equation

$$
u_{t}+u u_{x}=\varepsilon u_{x x}
$$

where $x \in X \subseteq \mathbb{R}, t \geqslant 0$, and $u: X \times \mathbb{R}^{+} \rightarrow \mathbb{R}$. The parameter $\varepsilon$ is typically referred to as the viscosity due to the connection between this equation and the stydy of fluid dynamics. When $\varepsilon>0$ it is often reffered to the viscous Burgers equation, and when $\varepsilon=0$, it is often reffered to the inviscid Burgers equation. Burgers equation was proposed as a model of turbulent fluid motion by J.M. Burgers in a series of sevral articles. It is one of the most important PDEs in the theory of non linear consevation laws. she combining

Received 2019-05-30; accepted 2019-07-12; published 2020-03-02.

2010 Mathematics Subject Classification. 35L99, 58J45.

Key words and phrases. non standard analysis; viscid and inviscid Burgers equation; N wave shock; periodic wave shock.

(C)2020 Authors retain the copyrights of their papers, and all open access articles are distributed under the terms of the Creative Commons Attribution License. 
both nonlinear propagation effects and diffusive effect. This equation is the approximation for the onedimensional propagation of weak shock waves in a fluid. It can also be used in the description of the variation in vehicle density in highway traffic. Burgers introduced the equation to describe the behavior of shock waves, traffic flow and acoustic transmission. This equation plays a relevant role in many different areas of the mathematical physics, specially in Fluid Mechanics. Moreover the simplicity of its formulation, in contrast with the Navier-Stokes system, makes of the Burgers equation a suitable model equation to test different numerical algorithms and results of a varied nature [2], [3]. If the viscous term is null, the remaining equation is hyperbolic this is the inviscid Burgers equation

$$
u_{t}+u u_{x}=0
$$

If the viscous term is dropped from the Burgers equation, discontinuities may appear in finite time, even if the initial condition is smooth they give rise to the phenomen of shock waves with important application in physics [10]. This properties make Burgers equation a proper model for testing numerical algorithms in flows where severe gradients or shocks are anticipated [1], [16], [18]. Recently, Kunjan and Twinkle [14] used mixture of new integral transform and Homotopy Perturbation Method to find the solution of Bugers equation arising in the longitudinal dispersion phenomenon in fluid flow through porous media. Discretization methods are well-known techniques for sollving Burgers equation. Ascher and Mclachlan established many methods as multisymplectic box sheme. Olayiwola, Gbolagade and Akinpelu [12] also presented the modified variational iteration method for the numerical solution of generalized Burgers-Huxley equation. For the boundary value problem, Sinai [16] is interested to the initial condition case: null on $\mathbb{R}_{-}$, and Brownian on $\mathbb{R}_{+}$. She, Aurell and Frich [15] with a numerical calculs particularly examine the initial conditions of Brownian fractionnair type. To study the initial value problem of Burgers equation, classical methods are based on search of solutions of the reduiced problem to deduce existence and asymptotic behaviour of the solutions as $\varepsilon$ tends to 0 , the passage of the limit is very complicated, but in general the limit exist and it's a solution for the reduced problem (when $\varepsilon=0$ ). For $\varepsilon$ small the solution $u(x, t)$ is approximated by this limit [10]. Other methods are based on a weak formulation of burgers equation seen as a conservation law satisfied on each of the computational domain called cell or finite volume. Stochastic particle method is so used for with different initial conditions. In particular we will take the same initial conditions that we took for the inviscid.

This paper completes recent works on the study of boundary value problems of Burgers equations for different initial conditions [2]- [4]. In the presented work, our general purpose is to describe the asymptotic behavior of solutions in boundary value problems with a small parameter $\varepsilon$ and to discuss in particular the cases of $\mathrm{N}$ wave and periodic wave shocks with new techniques infinitesimal of Non-Standard Analysis. We can conclude that the solution of the Cauchy problem of inviscid equation in each case is infinitely close to the solution of he Cauchy problem of viscid equation as $\varepsilon$ is a parameter positif sufficiently small. We 
introduce the infinitesimal techniques to give a simple formulation for the asymptotic behaviour. It is worth noting that our contribution is an elegant combination of infinitesimal techniques of Non-Standard Analysis and the Van Den Berg method [2], [3], [17].

Historically the subject non standard was developed by Robinson, Reeb, Lutz and Gose [11]. The Nonstandard perturbation theory of differential equations, which is today a well-established tool in asymptotic theory, has its roots in the seventies, when the Reebian school (see [11], [17]) introduced the use of NonStandard Analysis into the field of perturbed differential equations. Our goal in this paper is to generalize these techniques on PDE.

The paper is organised as follows: Section 2 concerns the boundary value problems of inviscid Burgers equation, we start with the Fitting discontinuous shock then we describe the asymptotic behaviour of solutions for this problem in the $\mathrm{N}$ wave and periodic wave cases. Section 3 concerns the boundary value problems of viscid Burgers equation, it contains basic preliminaries results and deals with our main results about $\mathrm{N}$ wave and periodic wave shock cases and its proof, we present it in a non standard form.

\section{Inviscid Burgers EquATION}

We will focus first on equation (1.2). Specifically, we will deal with the initial value problem

$$
\begin{cases}u_{t}+u u_{x}=0 & , \forall x \in \mathbb{R}, t>0 \\ u(\xi, 0)=f(\xi) & , t=0\end{cases}
$$

As it as has been suggested previously, although (2.1) seems to be a very innocent problem a priori it hides many unexpected phenomena. This problem does not admit the regular solutions but some weak solutions with certain regularity exist.The Burgers equation on the whole line is known to possess traveling waves solutions. Using the characteristic method, the solution of the problem (2.1) may be given in a parametric form

$$
\left\{\begin{array}{l}
u=f(\xi) \\
x=\xi+f(\xi) t
\end{array}\right.
$$

and shocks must be fitted in such that

$$
U=\frac{1}{2}\left(u_{1}+u_{2}\right)=\frac{1}{2}\left(f\left(\xi_{1}\right)+f\left(\xi_{2}\right)\right.
$$

Where $f: R \rightarrow R$. is a standard continuous function. $\xi_{1}$ and $\xi_{2}$ are the values of $\xi$ on the two sides of the shock [6].

According to $(2.2)$, the solution at time $t$ is obtained from the initial profile $u=f(\xi)$ by translating each point a distance $f(\xi) t$ to the right. The shock cuts out the part corresponding to $\xi_{2} \geq \xi \geq \xi_{1}$. If the discontinuity line it is a straight line chord betewen the points $\xi=\xi_{1}$ and $\xi=\xi_{2}$ on the curve $f(\xi)$. Moreover since areas are preserved under the mapping, the equal area property still holds. The chord on 
the $f$ curve cuts off lobes of equal area. The shock determination can then be describe entirely on the fixe $f(\xi)$ curve by drawing all the chords with the equal area propperty can be written analytically as

$$
\frac{1}{2}\left\{\left(f\left(\xi_{1}\right)+f\left(\xi_{2}\right)\right\}\left(\xi_{1}-\xi_{2}\right)=\int_{\xi_{2}}^{\xi_{1}} f(\xi) d \xi\right.
$$

This is the differential equation for the shock line chord wich verifies the entropic condition such as [6]. Since the left hand side is the area under the chord and the right hand side is the area under the $f$ curve. If the shock is at $x=s(t)$ at time $t$, we also have

$$
\begin{aligned}
& s(t)=\xi_{1}+f\left(\xi_{1}\right) t \\
& s(t)=\xi_{2}+f\left(\xi_{2}\right) t
\end{aligned}
$$

From (2.5) and (2.6), we have

$$
t=\frac{\xi_{1}-\xi_{2}}{f\left(\xi_{1}\right)-f\left(\xi_{2}\right)}
$$

2.1. Single Hump. To describe the solutions of the problem (2.1), we assume that the initial condition $f$ verify the following assumptions :

$\left(H_{1}\right): f$ is equal to a constant $u_{0}$ outside the range $0<\xi<L$.

$\left(H_{2}\right): f(\xi)>u_{0}$ in the range.

The theorem bellow gives the assymptotic behavior to the solution behind the shock and at the shock.

Theorem 2.1. Suppose that $\left(H_{1}\right)$ and $\left(H_{2}\right)$ are satisfied, the solution of the problem (2.1) is given by $(2.2)$ with $0<\xi<\xi_{2}$ and the asymptotic form is

$$
u \sim \frac{x}{t}, \text { for } u_{0} t<x<u_{0} t+\sqrt{2 A t}
$$

Proof. We consider the problem (2.1) and we suppose that $\left(H_{1}\right)$ and $\left(H_{2}\right)$ are satisfied. Equation (2.4) may be written as

$$
\frac{1}{2}\left\{\left(f\left(\xi_{1}\right)+f\left(\xi_{2}\right)-2 u_{0}\right\}\left(\xi_{1}-\xi_{2}\right)=\int_{\xi_{1}}^{L}\left(f(\xi)-u_{0}\right) d \xi\right.
$$

As time goes on $\xi_{1}$ increase and eventually exceed $L$. At this stage $f\left(\xi_{1}\right)=u_{0}$ and the shock is moving into the constant region $u=u_{0}$. The function $\xi_{1}(t)$ can then be eliminated for we have.

$$
\frac{1}{2}\left(f\left(\xi_{2}\right)-u_{0}\right)\left(\xi_{1}-\xi_{2}\right)=\int_{\xi_{2}}^{L}\left(f(\xi)-u_{0}\right) d \xi \quad, \quad t=\frac{\xi_{1}-\xi_{2}}{f\left(\xi_{2}\right)-u_{0}}
$$

There for

$$
\frac{1}{2}\left(f\left(\xi_{2}\right)-u_{0}\right)^{2} t=\int_{\xi_{2}}^{L}\left(f(\xi)-u_{0}\right) d \xi
$$


At this stage the shock position and the value of $u$ just behind the shock are given by

$$
\left\{\begin{array}{l}
u=f\left(\xi_{2}\right) \\
s(t)=\xi_{2}+f\left(\xi_{2}\right) t
\end{array}\right.
$$

where $\xi_{2}$ satisfies $(2.11)$. As $t$ is infinitely large we have $\xi_{2}$ infinitesimal and $f\left(\xi_{2}\right)$ approach $u_{0}$, hence the equation for $\xi_{2}(t)$ takes the limiting form

$$
\frac{1}{2}\left(f\left(\xi_{2}\right)-u_{0}\right)^{2} t \sim A
$$

where

$$
A=\int_{0}^{L}\left(f(\xi)-u_{0}\right) d \xi
$$

is the area of the hump above the undisturbed value $u_{0}$. We have $\xi_{2}$ infinitesimal and

$$
f\left(\xi_{2}\right) \sim u_{0}+\sqrt{2 A / t}
$$

Therefore the asymptotic formulas for $s(t)$ and $u$ in $(2.12)$ are

$$
\begin{aligned}
& s(t) \sim u_{0} t+\sqrt{2 A t} \\
& u-u_{0} \sim \sqrt{2 A / t}
\end{aligned}
$$

at the shock. The shock curve is asymptotically parabolic. The solution behind the shock is given by (2.2) with $0<\xi<\xi_{2}$. Since $\xi_{2}$ is small enouhg as $t$ is small enouhg, all the relevant values of $\xi$ also small enouhg and the asymptotic form is

$$
u \sim \frac{x}{t}, \text { for } u_{0} t<x<u_{0} t+\sqrt{2 A t}
$$

The asymptotic solution and the corresponding $(x, t)$ diagram are shown in Figure 1.

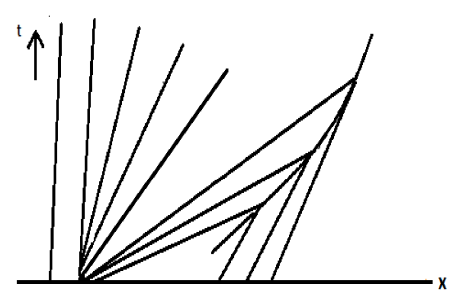

Figure 1. The Asymptotic triangular wave 


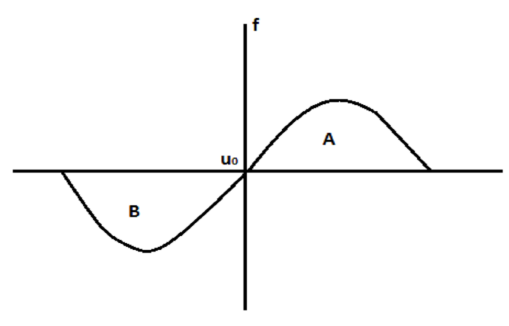

Figure 2. The profile of the initial condition in the $\mathrm{N}$ wave case

2.2. $\mathbf{N}$ Wave. Other problem can be worked out in similar way, one important case is when $f(\xi)$ has a positive and a negative phase about an undisturbed value $u_{0}$ as in Figure 2.

There are now two shocks, corresponding to the two compression phases at the front and at the back where $f^{\prime}(\xi)<0$. The families of chords for each are shown in the figure. As $t$ is infinitely large, the pair $\left(\xi_{2}, \xi_{1}\right)$ for the front shock approach $(0, \infty)$, where as for the rear shock $\left(\xi_{2}, \xi_{1}\right)$ approach $(-\infty, 0)$. Asymptotically the front shock is

$$
s \simeq u_{0} t+\sqrt{2 A t}
$$

and the jump of $u$ is

$$
u-u_{0} \sim \sqrt{2 A / t}
$$

where $A$ is the area of the $f$ curve above $u=u_{0}$. The rear shock has

$$
x \sim u_{0} t-\sqrt{2 B t}
$$

$$
u-u_{0} \sim-\sqrt{2 B / t}
$$

where $B$ is the area below $u=u_{0}$. The solution between the shocks is again asymptotically

$$
u \sim \frac{x}{t}, \quad u_{0} t-\sqrt{2 B t}<x<u_{0} t+\sqrt{2 A t}
$$

The asymptotic form and the $(x, t)$ diagramm are shown in Figure 3 and Figure 4.

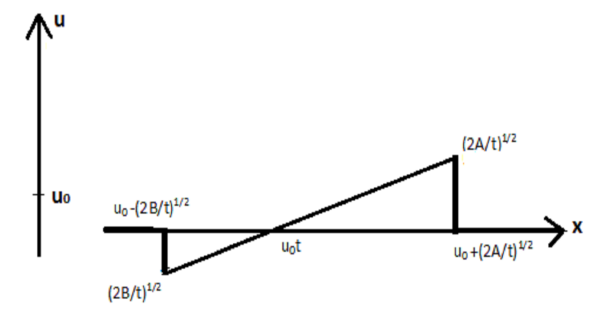

FIGURE 3. Shock construction for $\mathrm{N}$ wave 


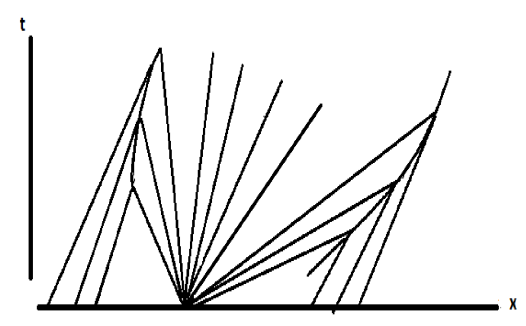

FIgURE 4 . The asymptotic $\mathrm{N}$ wave

2.3. Periodic Wave. Another intersting problem is that of an initial distribution

$$
f(\xi)=u_{0}+a \sin \frac{2 \pi \xi}{\lambda}
$$

In this case, the shock equations (2.4) simplify considerably for all times $t$. Consider one period $0<\xi<\lambda$ as in Figure 5. Relations (2.4) becomes

$$
\left(\xi_{1}-\xi_{2}\right) \sin \frac{\pi}{\lambda}\left(\xi_{1}+\xi_{2}\right) \cos \frac{\pi}{\lambda}\left(\xi_{1}-\xi_{2}\right)=\frac{\lambda}{\pi} \sin \frac{\pi}{\lambda}\left(\xi_{1}-\xi_{2}\right) \sin \frac{\pi}{\lambda}\left(\xi_{1}+\xi_{2}\right)
$$

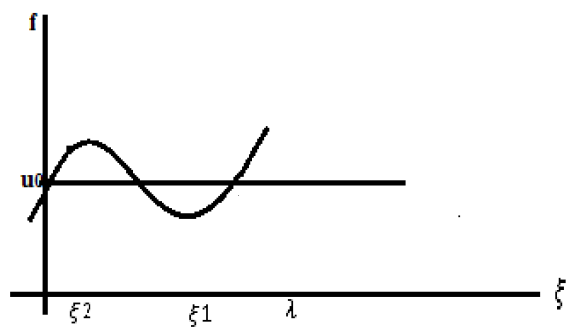

FIGURE 5. Shock construction for a periodic wave

and the relevant choice is the trivial one

$$
\sin \frac{\pi}{\lambda}\left(\xi_{1}+\xi_{2}\right)=0 \quad \text { that is } \quad \xi_{1}+\xi_{2}=\lambda
$$

From the difference and sum of (2.5) and (2.6) we have

$$
\begin{gathered}
t=\frac{\xi_{1}-\xi_{2}}{2 a \sin \frac{\pi}{\lambda}\left(\xi_{1}-\xi_{2}\right)} \\
s=u_{0} t+\frac{\lambda}{2}
\end{gathered}
$$

Respectively. The discontinuity in $u$ at the shock is

$$
\begin{aligned}
u_{2}-u_{1} & =a \sin \frac{2 \pi \xi_{1}}{\lambda}-a \sin \frac{2 \pi \xi_{2}}{\lambda} \\
& =2 a \sin \frac{\pi}{\lambda}\left(\xi_{1}-\xi_{2}\right)
\end{aligned}
$$


If we introduce

$$
\xi_{1}-\xi_{2}=\frac{\lambda \theta}{\pi} \quad, \quad \xi_{1}+\xi_{2}=\lambda
$$

we have

$$
\begin{gathered}
t=\frac{\lambda}{2 \pi a} \cdot \frac{\theta}{\sin \theta} \\
s=u_{0} t+\frac{\lambda}{2} \\
\frac{u_{2}-u_{1}}{u_{0}}=\frac{2 a}{u_{0}} \sin \theta
\end{gathered}
$$

The shock has constant velocity $u_{0}$ and this result could have been deduced in advance from the symmetry of the problem. The shock starts with zero strength corresponding to $\theta=0$ at time $t=\lambda / 2 \pi a$. It reaches a maximum strength of $2 a / u_{0}$ for $\theta=\pi / 2, t=\lambda / 4 a$ and decays ultimately with $\theta$ approach $\pi$, when $t$ is infinitely large

$$
\frac{u_{2}-u_{1}}{u_{0}} \sim \frac{\lambda}{u_{0} t}
$$

It is interesting that the final decay formula does not even depend explicitly on the amplitude $a$, however the condition for its application is $t>>\lambda / a$. For any periodic sinusoidal $f(\xi)$ or not $\xi_{1}-\xi_{2} \rightarrow \lambda$ as $t$ infinitely large; thence from (2.7)

$$
\frac{u_{2}-u_{1}}{u_{0}}=\frac{f\left(\xi_{2}\right)-f\left(\xi_{1}\right)}{u_{0}} \sim \frac{\lambda}{u_{0} t}
$$

Between successive shocks, the solution for $u$ is linear in $x$ with slope $1 / t$ as before, and the asymptotic form of the entire profile is the Sawtooth shown in Figure 6.

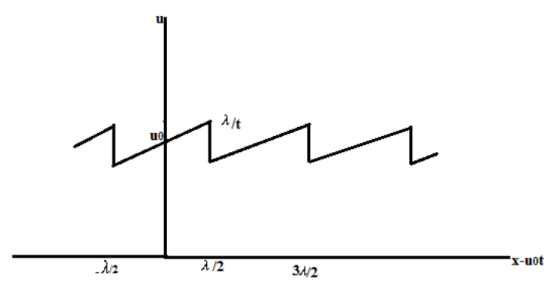

Figure 6. Asymptotic form of a periodic wave

\section{Viscid Burgers Equation}

In this section we shall present and prove our main results, we discuss the $\mathrm{N}$ wave and periodic wave cases in the boundary value problem of viscid Burgers equation

$$
\begin{cases}u_{t}+u u_{x}=\varepsilon u_{x x} & , \forall x \in \mathbb{R}, \quad t>0 \\ u(\xi, 0)=f(\xi) & , t=0\end{cases}
$$

Before going further in this cases we need the following proposition and lemma : 
3.1. The Cole-Hopf Transformation. Cole and Hopf noted the remarkable result [9] that the viscid Burgers equation (1.1) may be reduced to the linear Heat equation

$$
\varphi_{t}=\varepsilon \varphi_{x x}
$$

by the non linear transformation

$$
u=-2 \varepsilon[\log \varphi]_{x}
$$

It is again convenient to do the transformation in two steps. Firstly are introduced

$$
u=\psi_{x}
$$

So that (1.1) may be integrated to

$$
\psi_{t}+\frac{1}{2} \psi_{x}^{2}=\varepsilon \psi_{x x}
$$

Then we introduce

$$
\psi=-2 \varepsilon[\log \varphi]
$$

To obtain (3.2).

The non linear transformation just eliminates the nonlinear term. The general solution of the Heat equation (3.2) is well known and can be handled by a variety of methods. The basic problem considered in section 2 is the initial value problem

$$
u=f(\xi), \text { at } t=0
$$

this is transformed by (3.3) to the initial value problem

$$
\varphi=\phi(x)=\exp \left\{-\frac{1}{2 \varepsilon} \int_{0}^{x} f(\eta) d \eta\right\} \quad, \quad t=0
$$

For the Heat equation, the solution for $\varphi$ is

$$
\varphi=\frac{1}{\sqrt{4 \pi \varepsilon t}} \int_{-\infty}^{+\infty} \phi(\eta) \exp \left\{-\frac{(x-\eta)^{2}}{4 \varepsilon t}\right\} d \eta
$$

Through (3.3), the solution for $u$ is

$$
u(x, t)=\frac{\int_{-\infty}^{+\infty} \frac{x-\eta}{t} e^{-G / 2 \varepsilon} d \eta}{\int_{-\infty}^{+\infty} e^{-G / 2 \varepsilon} d \eta}
$$

where

$$
G(\eta, x, t)=\int_{0}^{\eta} f(\nu) d \nu+\frac{(x-\eta)^{2}}{2 t}
$$


3.2. The Behavior of Solutions as $\varepsilon$ Small Enough. The behavior of the exact solution (3.6) is now considered as $\varepsilon$ is small enough. For $x, t$ and $f(x)$ are held fixed as $\varepsilon$ is small enough, the dominant contributions to the integrals in (3.6) come from the neighborhood of the stationary points of $G$. A stationary point is where

$$
\frac{\partial G}{\partial \eta}=f(\eta)-\frac{x-\eta}{t}=0
$$

Let $\eta=\xi(x, t)$ be such a point that is $\xi(x, t)$ is difined as a solution of

$$
f(\xi)-\frac{x-\xi}{t}=0
$$

The contribution from the neighborhood of a stationary point $\eta=\xi$ in an integral is given with the lemma 3.2 .

Lemma 3.1. (The Van. Den .Berg lemma 12). let $G$ be a standard function definied and increasing on $\left[0,+\infty\left[\right.\right.$ such that $G(v)=a v^{r}(1+\delta)$ for $v \simeq 0$ and $G(v)>m(v)^{q}$. Let $\varphi$ be an intern function definied on $] 0,+\infty\left[\right.$ such that $: \varphi(v)=b v^{s}(1+\delta)$ for $v \approx 0$ and such that $\forall d>0, \exists$ standard $k$ and $c$ such that : $|\varphi(v)|<k \exp (\cosh (v))$ for $v>d$. Then

$$
\int_{0}^{\infty} \varphi(v) \exp \left(-\frac{G(v)}{2 \varepsilon}\right) d v=\frac{b \Gamma\left(\frac{(s+1)}{r}\right)}{r a^{\frac{(s+1)}{r}}} \frac{1}{\left(\frac{1}{2 \varepsilon}\right)^{\frac{(s+1)}{r}}}
$$

where $a$ and $r$ are positifs standard, $m$ and $q$ are the both positifs. $\delta$ is a positif real small enough. $b$ and $s$ are standard, $b \neq 0$ and $s>-1$.

Lemma 3.2. (The Nonstandard formula of the method of steepest descents). Let $\varepsilon$ be a positif rael small enough and let $\varphi$ and $G$ be two standard functions such that: $G$, is a $C^{2}$ class function verifies the lemma 3.1, and admits on the $\xi$ point an unique absolute minimum $\left(G^{\prime}(\xi)=0\right.$ et $\left.G^{\prime \prime}(\xi)>0\right)$. $\varphi(\xi) \neq 0$, it is $S$ continuous on $\xi$ and satisfies the conditions of the lemma 3.1 in the two sens. Then

$$
\int_{-\infty}^{+\infty} \varphi(\eta) \cdot e^{-G / 2 \varepsilon} d \eta=\varphi(\xi) \frac{\sqrt{4 \pi \varepsilon}}{\sqrt{G^{\prime \prime}(\xi)}} \cdot e^{-G / 2 \varepsilon}(1+\delta)
$$

$\delta$ is a positif real small enough.

Proof. Suppose first that there is only one stationary point $\xi(x, t)$ wich satisfies $(3.8)$ then

$$
\begin{gathered}
\int_{-\infty}^{+\infty} \frac{x-\eta}{t} e^{-G / 2 \varepsilon} d \eta=\frac{x-\eta}{t} \frac{\sqrt{4 \pi \varepsilon}}{\sqrt{G^{\prime \prime}(\xi)}} e^{-G / 2 \varepsilon}(1+\delta) \\
\int_{-\infty}^{+\infty} e^{-G / 2 \varepsilon} d \eta=\frac{\sqrt{4 \pi \varepsilon}}{\sqrt{G^{\prime \prime}(\xi)}} e^{-G / 2 \varepsilon}(1+\delta)
\end{gathered}
$$

and in (3.6) we have

$$
u \simeq \frac{x-\xi}{t}
$$


where $\xi(x, t)$ is defined by (3.8). This asymptotic solution may be rewritten as

$$
\left\{\begin{array}{l}
u=f(\xi) \\
x=\xi+f(\xi) t
\end{array}\right.
$$

It is exactly the solution of (2.1) witch was discussed in section 2 . The stationary point $\xi(x, t)$ becomes the characteristic variable.

\subsection{The Main Results.}

3.3.1. $N$ Wave. Another example, we consider is more easily derived by choosing appropriate solutions for $\varphi$ to satisfy the Heat equation and then substituting in (1.4) to obtain $u$ as a rough qualitative guide to the appropriate choice. The profile of $u$ will be some thing like $\varphi_{x}$. To obtain an $\mathrm{N}$ wave of $u$. We choose the source solution of the Heat equation for $\varphi$

$$
\varphi=1+\sqrt{\frac{a}{t}} \cdot e^{\left(-x^{2} / 4 \varepsilon t\right)}
$$

Since $\varphi$ has a $\delta$ function behavior as $t$ is infinitesimal, this is a little hard to interpret as an initial value problem on $u$. However, for any $t>0$, it has the form shown in Figure 2 with a positive and a negative phase and we may take the profile at any $t=t_{0}$ to be the initial profile. It should typical of all $\mathrm{N}$ wave solutions.

Theorem 3.1. (I). Assume that the initial data $f$ has a profile shown in Figure 2, the problem (1.3) admit an unique solution for $t>0$ given by

$$
u \sim\left\{\begin{array}{cr}
\frac{x}{t}, & -\sqrt{2 A t}<x<\sqrt{2 A t} \\
0, & |x|>\sqrt{2 A t}
\end{array}\right.
$$

where $A=\int_{-\infty}^{+\infty}\left(f(x)-u_{0}\right) d x$.

(II). Such solution present $N$ wave chocks, and for $\varepsilon$ small enough, this solution is infinitely close to the solution of the inviscid problem (1.4) giveb in section 2.

Proof. To obtain an $\mathrm{N}$ wave for $u$, we choose the source solution of the Heat equation for $\varphi$ given by (3.13). Then the corresponding solution for $u$ is

$$
u=-2 \varepsilon \frac{\varphi_{x}}{\varphi}=\frac{x}{t} \cdot \frac{\sqrt{\frac{a}{t}} \cdot \exp \left(-\frac{x^{2}}{4 \varepsilon t}\right)}{1+\sqrt{\frac{a}{t}} \cdot \exp \left(-\frac{x^{2}}{4 \varepsilon t}\right)}
$$

Since $\varphi$ has a $\delta$ function behavior as $t$ is infinitely small, this is a little hard to interpret as an initial value problem on $u$. However for any $t>0$ it has the form shown in Fig.6 with a positive and negative phase 
and we may take the profile at any $t=t_{0}$ to be the initial profile. It should be typical of all $\mathrm{N}$ Wave solution. The area under the positive phase of the profile is

$$
\int_{0}^{+\infty} u d x=-2 \varepsilon[\log \varphi]_{0}^{\infty}=2 \varepsilon \log \left[1+\sqrt{\frac{a}{t}}\right]
$$

The positive phase is infinitely small when $t$ is infinitely large. If the value of (3.14) at the initial time $t_{0}$ is denoted by $A$ we may introduce a Reynolds number

$$
R_{0}=\frac{A}{2 \varepsilon}=\log \left(1+\sqrt{\frac{a}{t_{0}}}\right)
$$

But as time goes on the effective, Reynolds number will be

$$
R(t)=\frac{1}{2 \varepsilon} \int_{0}^{+\infty} u d x=\log \left(1+\sqrt{\frac{a}{t}}\right)
$$

and this is infinitely small as $t$ is infinitely large.

If $R_{0}>>1$, We may expect the "Inviscid Theory" of (2.1) and (2.2) to be a good approximation for some time but as $t$ is infinitely large , $R(t)$ is eventually become dominant. In terms of $R_{0}$ and $t_{0}, a=$ $t_{0}\left(\exp \left(R_{0}\right)-1\right)$. Hence $(3.14)$ may be written.

$$
u=\frac{x}{t} \cdot\left\{1+\sqrt{\frac{t}{t_{0}}} \frac{\exp \left(\frac{x^{2}}{4 \varepsilon t}\right)}{\exp \left(R_{0}\right)-1}\right\}^{-1}
$$

and For $R_{0}>>1$ ( corresponding to $t_{0}<<0$ ), we have

$$
\exp \left(R_{0}\right)-1 \sim \exp \left(R_{0}\right)
$$

and

$$
\frac{\exp \left(\frac{x^{2}}{4 \varepsilon t}\right)}{\exp \left(R_{0}\right)-1} \sim \exp \left(\frac{x^{2}}{4 \varepsilon t}-R_{0}\right) \sim \exp R_{0}\left(\frac{x^{2}}{2 A t}-1\right) .
$$

Or $u$ in (3.17) may be approximated by

$$
u=\frac{x}{t} \cdot\left\{1+\sqrt{\frac{t}{t_{0}} \exp \left[R_{0}\left(\frac{x^{2}}{2 A t}-1\right)\right]}\right\}^{-1}
$$

For $x$ and $t$. Now for fixed $t$ limited and $R_{0}$ infinitely large we have

$$
u \sim \begin{cases}\frac{x}{t}, & \text { if } \frac{x^{2}}{2 A t}-1<0, \quad-\sqrt{2 A t}<x<\sqrt{2 A t} \\ 0, & \text { if } \frac{x^{2}}{2 A t}-1<0, \quad|x|>\sqrt{2 A t}\end{cases}
$$

This is exactly the inviscid solution.

However, for any fixed $a$ and $\varepsilon$ we see directely from (3.15) [and it may be verified also from (3.19)] that

$$
u \sim \frac{x}{t} \cdot \sqrt{\frac{a}{t}} \exp \left(-\frac{x^{2}}{4 \varepsilon t}\right)
$$


as $t$ is infinitely large. This is the dipole solution of the Heat equation. The diffusion dominates the nonlinear term in the final decay. It should be remembered though,that this final period of decay is for extremely large times; the Inviscid theory is adequate for most of the interesting range.

3.3.2. Periodic Wave. A periodic solution may be obtained by taking for $\varphi$ a distribution of Heat sources spaced a distance $\lambda$ apart. Then

$$
\varphi=\frac{1}{\sqrt{4 \pi \varepsilon t}} \sum_{n=-\infty}^{\infty} \exp \left\{-\frac{(x-n \lambda)^{2}}{4 \varepsilon t}\right\}
$$

Theorem 3.2. (I). Assume that the initial data $f$ has a profile shown in Fig 5 . When $\lambda^{2} / 4 \varepsilon t>>1$, the problem (3.1) admit an unique solution for $t>0$ given by

$$
u \sim \frac{x-m \lambda}{t}, \quad(m-1 / 2) \lambda<x<(m+1 / 2) \lambda
$$

(II). Such solution is the periodic wave chock, and for $\varepsilon$ small enough, this solution is infinitely close to the solution of the inviscid problem (2.1) given in section 2.

Proof. To obtain a periodic wave for $u$, we choose for $\varphi$ a distribution of Heat sources spaced a distance $\lambda$ given by (3.14). Then the corresponding solution for $u$ is

$$
u=-2 \varepsilon \frac{\varphi_{x}}{\varphi}=\frac{\sum_{n=-\infty}^{\infty}\left(\frac{x-n \lambda}{t}\right) \exp \left\{-\frac{(x-n \lambda)^{2}}{4 \varepsilon t}\right\}}{\sum_{n=-\infty}^{\infty} \exp \left\{-\frac{(x-n \lambda)^{2}}{4 \varepsilon t}\right\}}
$$

For $\lambda^{2} / 4 \varepsilon t>>, 1$ this implies that $\sqrt{\varepsilon t}<<\lambda / 2$, and $\sum_{n=-\infty}^{\infty}=2 \sum_{n=0}^{\infty}$, then for $n=m$ we have

$$
\frac{(x-n \lambda)^{2}}{4 \varepsilon t}=\frac{(x-m \lambda)^{2}}{\varepsilon t}<<1
$$

wich gives $|x-m \lambda|<<\sqrt{\varepsilon t}$. and the exponential with the minimum value of $(x-n \lambda)^{2} / 4 \varepsilon t$ will dominate over all the others. Therefore the term witch will dominate for

$$
(m-1 / 2) \lambda<x<(m+1 / 2) \lambda
$$

and (3.15) is approximately

$$
u \sim \frac{x-m \lambda}{t}, \text { for }(m-1 / 2) \lambda<x<(m+1 / 2) \lambda
$$

This is a sawtooth wave with a periodic set of shocks a distance $\lambda$ apart, and $u$ jumps from $-\lambda / 2 t$ to $\lambda / 2 t$ at each shock. The result agrees with he inviscid solution given by (2.20).

Corollary 3.1. If $\frac{\lambda^{2}}{4 \varepsilon t}<<1$, the initial data can be expanded in a Fourier series as

$$
\varphi=\frac{1}{\lambda}\left\{1+2 \sum_{n=1}^{\infty} \exp \left(-\frac{4 \pi^{2} n^{2}}{\lambda^{2}} \varepsilon t\right) \cos \frac{2 \pi n x}{\lambda}\right\}
$$


and for $t>0$, we have

$$
u \sim \frac{8 \pi \varepsilon}{\lambda} \exp \left\{-\frac{4 \pi^{2} \varepsilon t}{\lambda^{2}}\right\} \sin \frac{2 \pi x}{\lambda}
$$

This is a solution of the Heat equation.

Proof. To study the Final decay $\left(\lambda^{2} / 4 \varepsilon t\right)<<1$, we may use an alternative form of the solution. The expression (3.14) is periodic in $x$, and in the interval: $-\lambda / 2<x<\lambda / 2, \varphi \rightarrow \delta(x)$, as $t$ is infinitely small. The initial condition can be expanded in a Fourier series as

$$
\phi(x)=\frac{1}{\lambda}\left\{1+2 \sum_{n=1}^{\infty} \cos \frac{2 \pi n x}{\lambda}\right\}
$$

and the corresponding solution of the Heat equation for $\varphi$ is

$$
\varphi=\frac{1}{\lambda}\left\{1+2 \sum_{n=1}^{\infty} \exp \left(-\frac{4 \pi^{2} n^{2}}{\lambda^{2}} \varepsilon t\right) \cos \frac{2 \pi n x}{\lambda}\right\}
$$

It may be verified directly that this is the Fourier series of .(3.14). In this form

$$
u=-2 \varepsilon \frac{\varphi_{x}}{\varphi}=\frac{\frac{8 \pi \varepsilon}{\lambda} \sum_{n=1}^{\infty} n \exp \left(-\frac{4 \pi^{2} n^{2}}{\lambda^{2}} \varepsilon t\right) \sin \frac{2 \pi n x}{\lambda}}{1+2 \sum_{n=1}^{\infty} \exp \left(-\frac{4 \pi^{2} n^{2}}{\lambda^{2}} \varepsilon t\right) \cos \frac{2 \pi n x}{\lambda}}
$$

when $\frac{\varepsilon t}{\lambda^{2}}>>1$, the term with $n=1$ dominate the series and we have

$$
u \sim \frac{8 \pi \varepsilon}{\lambda} \exp \left\{-\frac{4 \pi^{2} \varepsilon t}{\lambda^{2}}\right\} \sin \frac{2 \pi x}{\lambda}
$$

This is a solution of

$$
u_{t}=\varepsilon u_{x x}
$$

and the diffusion dominate in the ultimate decay.

\section{Conclusion}

This paper completes recent works on the study of boundary value problems of Burgers equations for different initial conditions [2]- [4]. Our general purpose is to describe the asymptotic behavior of solutions in boundary value problem with a small parameter $\varepsilon$ and to discuss in particular the $\mathrm{N}$ wave shocks and Periodic wave shocks cases. The originality of this work consists in introducing new infinitesimal techniques of Non-Standard Analysis. We can conclude that the solution of the Cauchy problem of inviscid equation in each case is infinitely close to the solution of he Cauchy problem of viscid equation as $\varepsilon$ is a parameter positif sufficiently small. We introduce the infinitesimal techniques to give a simple formulation for the asymptotic behavior. It is worth noting that our contribution is an elegant combination of infinitesimal techniques of non standard analysis and the Van Den Berg method [2], [3], [17]. 
Conflicts of Interest: The author(s) declare that there are no conflicts of interest regarding the publication of this paper.

\section{REFERENCES}

[1] Alexy, Samokhin, Gradient catastrophes and saw tooth solution for a generalized Burgers equation on an interval, J. Geom. Phys. 85 (2014), 177-184.

[2] S. Bendaas, The Asymptotic Behavior of Viscid Burgers solution in the N wave shock case. A new Approach, Asian J. Math. Computer Res. 12 (3) (2016), 221-232.

[3] S. Bendaas, Confluence of Shocks in Burgers Equation. A new Approach, Int. J. Differ. Equ. Appl. 14 (2015), 369-382.

[4] S. Bendaas, Boundary value problems for Burgers equations through Nonstandard Analysis, Appl. Math. 6 (2015), 10861098.

[5] S. Bendaas, L'équation de Burgers avec un terme dissipatif. Une approche non standard, Analele universitatii Oradea, Fasc. Math. 15 (2008), 239-252.

[6] S. Bendaas, Quelques applications de l'A.N.S aux E.D.P, Thèse de Doctorat. Université de Haute Alsace, France, (1994).

[7] J. M. Burgers, The non linear diffusion equation asymptotic solution and satatistical propblems, Reidel, 1974.

[8] D. Euvrard, Résolution numérique des équations aux dérivées partielles, Différences finies, éléments finis/Masson. Paris, Milan, Barcelone, Mexico (1989).

[9] E. Hopf, The partial differential equation $u_{t}+u u_{x}=\nu u_{x x}$, Commun. Pure Appl. Mech. 3 (1950), 201-230.

[10] S. Kida, Asymptotic properties of Burgers turbulence, Journal of Fluid mechanics, 93(1979), 337-377.

[11] R. Lutz and M. Goze, Non Standard Analysis. A. Practical Guide with application Lectures, Notes in Math. N 861. Springer Verlag, Berlin (1981).

[12] M.O. Olayiwola, A.W. Gbolagade and F.O. Akinpelu, Numerical solution of generalized Burger's-Huxley equation by modified variational iteration method, J. Nigerian Assoc. Math. Phys. 17 (2010), 433-438.

[13] A. V. Samokhin, Evolution of initial data for Burgers equation with fixed boundary values, Sci. Herald MSTUCA, 194 (2013), 63-70 (In Russian).

[14] K. Shah and T. Singh, A solution of the Burger's equation arising in the longitudinal dispersion phenomena in fluid flow through porous media by mixture of new integral transform and homotopy perturbation method, J. Geosci. Environ. Protect. 3 (2015), 24-30.

[15] Z. S. She, E. Aurell and U. Frisch, The Inviscid Burgers equation with initial data of brownien type, Commun. Math. Phys. 148 (1992), 623-641.

[16] Ya. G. Sinai, Statistics of shocks in solutions of inviscid Burgers equation, Commun. Math. Phys. 148 (1992), 601-621.

[17] I. Van Den Berg, Non Standard Asymptotic Analysis Lectures, Notes in Math. Vol. 1249. Springer Verlag.

[18] A. M. Wazwaz, Travelling wave solution of generalized forms of Burgers, Burgers-KdV and Burger's-Huxley equations, App. Math. Comput. 169 (2005), 639-656. 